\title{
Signal Operation with DFT Process in Real Time
}

\author{
Li Xiangnan \\ School of Information and Electronics, Beijing Institute of Technology, Beijing, 100081, China \\ E-mail: Ixn196768@163.com
}

Keywords: DFT, real time, sample storage, vertical vector

\begin{abstract}
A kind of DFT process used for signal operation in real time is proposed. The complicated environment for modern electronic counter and the appearance of new radar systems put forward a new lesson to sub-collect the signal and track the signal, especially in real time. With the background of high speed, this thesis researched the acquisition in some aspect. It analyzed the DFT possibility of having the higher efficiency comparing with FFT and putted forward a kind of DFT processor construction. This method is proper for the fast process in order to get the real time DFT
\end{abstract}

\section{Introduction}

Usually, the operation system divides to independent sampling and DFT parts [1]-[2]. And the total quantity of DFT calculation which reaches $N^{*} N$ is too large for hardware realization. In the traditional FFT algorithm, the reduction of calculation leads shorter time, but it still wastes the sampling time for independent. In this paper the algorithm is not only based on the total quantity of calculation, but the quantity and unit time domain of each sampling cycle. As considered the cost of time, the process is along with the sampling and there is no need to storage the signals.

From the basic formula of N-point DFT, each sample signal is been utilized N times [4]. In this process it needs $\mathrm{N}$ times of operation which is a combination of multiplier and adder (CMA). The operation formula is shown below in (1).

$$
\begin{aligned}
X(k)=\sum_{l=1}^{N} S(x l) \cdot W^{l k} & =A(k)+i B(k), W=e^{-i \frac{2 \pi}{N}} \\
A(k) & =\sum_{l=1}^{N} S(x l) \cdot \cos \frac{2 \pi l k}{N} \\
B(k) & =\sum_{l=1}^{N} S(x l) \cdot \sin \frac{2 \pi l k}{N}
\end{aligned}
$$

Here $S(x l)$ is a matrix with limit values from the sampled signal. The operation contains 2 parts of (2) and (3).

\section{The basic flow}

From the formula above, the complex number of the DFT formula is separated in to cosine gene and sine gene. The operations of two functions are totally the same. Therefore, only cosine function is taken for example, and the algorithm of sine is similar [3]-[4].

For this cosine part,

$$
A(k)=\sum_{l=1}^{N} S(x l) \cdot \cos \frac{2 \pi l k}{N}
$$

Its matrix form with $\mathrm{N}$ point is

$$
\left[A_{k}\right]_{1 \times N}=\left[\cos \frac{2 \pi l k}{N}\right]_{N \times N} \cdot[S(x l)]_{1 \times N}
$$

The matrix of $N^{*} N$ can be written as vertical vector form shown below. Here $C_{i}$ is the sign for 
the matrix of gene vectors.

$$
\begin{aligned}
{\left[A_{k}\right]_{1 \times N} } & =S(x 1) \cdot\left[\cos \frac{2 \pi l \cdot 1}{N}\right]_{1 \times N}+\cdots S(x i) \cdot\left[\cos \frac{2 \pi l \cdot i}{N}\right]_{1 \times N}+\cdots S(x N) \cdot\left[\cos \frac{2 \pi l \cdot N}{N}\right]_{1 \times N} \\
& =S(x 1) \cdot C_{1}+\cdots S(x i) \cdot C_{i}+\cdots S(x N) \cdot C_{N}
\end{aligned}
$$

From the transformation of the matrix formula above, the calculation for $S(x i) \bullet C_{i}$ is the repeated utilization of single signal value $S(x i)$ processing only with the coefficient $C_{i}$ change. It starts from the sampling point $i$ to $i+1$ with each sampling cycle. That means in each sampling period, the matrix calculation of last sampling value and corresponding matrix vector should be finished.

The flow of time series is shown in Table 1 . Here $T_{s}$ is the sampling cycle, and the sign of * means the blank cell.

Table 1 Time series

\begin{tabular}{|c|c|c|c|c|}
\hline$T_{s 1}$ & $T_{s 2}$ & $\ldots T_{s i} \ldots$ & $T_{s N}$ & $*$ \\
\hline$*$ & $S(x 1) \bullet C_{1}$ & $S(x 2) \bullet C_{2}$ & $\ldots S(x) \bullet C_{i} \ldots$ & $S(x N) \bullet C_{N}$ \\
\hline
\end{tabular}

In fact, the process contains 2 parts in $T_{s}$. Fig. 1 shows the framework. Firstly, multiplying the $l$ th sample value with the $k$ th cosine vector, the result is the increment of frequency vector. Secondly, plus the $k$ th vector, it finishes the $\mathrm{N}$ times frequency calculation in $(l+1)$ th sampling time.

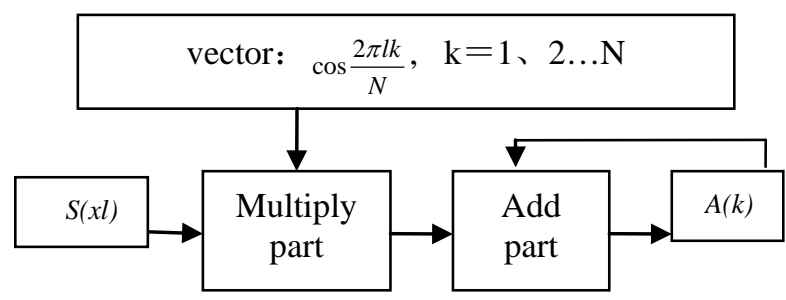

Fig. 1. The operation framework

The operation of this sampling signal should be finished during each sampling gap time, for Npoint DFT, $\mathrm{N}$ times multiply should be done in the time of $T_{s}$. So it needs to arrange the calculation time properly. In order to save time, Fig.2 takes more resource. It can be adjusted the flow of algorithm according to actual hardware, and be chosen the utilization of the time and hardware resource equally, in order to finish the Fourier transform by multi-channel.

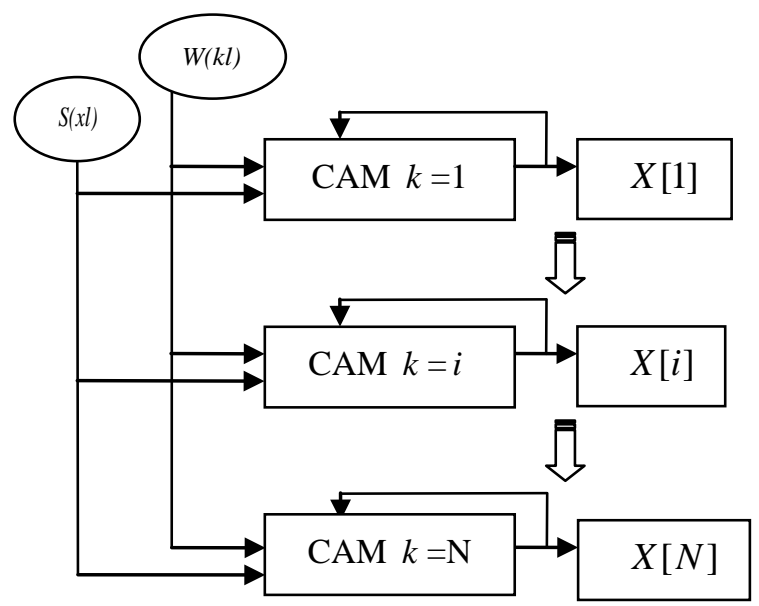

Fig. 2. The mode of multi-channel in a $T_{s}$ time 


\section{Analysis of result}

\section{A. Process of algorithm}

According to the analysis and method of the realization above, simulation will be done here. Take the signal below as an example to show the operation process. Selected by $\mathrm{N}=1024$, then the expression of series sampling signal is shown in (7) and Fig.3. Here are the points of frequencies, $f_{1}=10 e 6$ with 81 th, $f_{2}=20 e 6$ with 161 th, and $f_{s}=128 e 6$ with $0<n<1024$.

$$
x(n)=\sin \left(2 \pi n \cdot f_{1} / f_{s}\right)+0.5 \sin \left(2 \pi n \cdot f_{2} / f_{s}\right)
$$

The result of the algorithm of this paper to operate DFT is shown as Fig. 3. And Fig.4 shows the changes of the magnitude at corresponding point (81\&161) when it is $\mathrm{N}$ times of $T_{s}$ (here $T_{s}$ is $1 / \mathrm{fs}$ ).
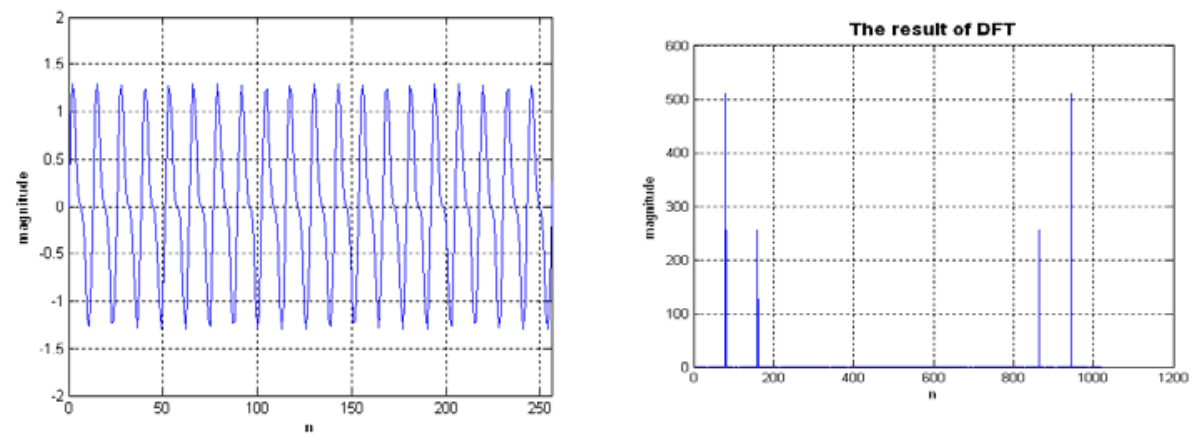

Fig. 3.The input signal and The results of DFT

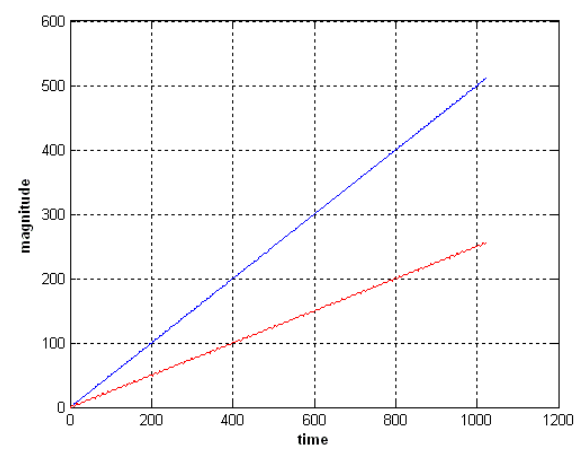

Fig. 4.The 81th and 161th points in time domain

B. Storage of the sample points and gene values

Compared to traditional FFT, this process does not need storage part for the sample signals as each sample point starts to be used when it reaches. After calculation during the sampling cycle, the value of this sample point can be covered by the next. This results with zero storage for sample. The storage amount of the exponential value of this algorithm is N/4, same as the rotate genes amount of traditional FFT which participates in compute.

\section{Result}

As a result, 2FSK signals are demodulated using this algorithm in Matlab shown in Fig. 6. It shows the advanced DFT process in real time. Here the input signals are combined with 2 frequencies [5], and each frequency has its own code ' 0 ' or ' 1 '. Under the DFT process, the relative point of each frequency shows the changes of the amplitude. After the distinguished by 1 bit, the results shows the demodulation code. 


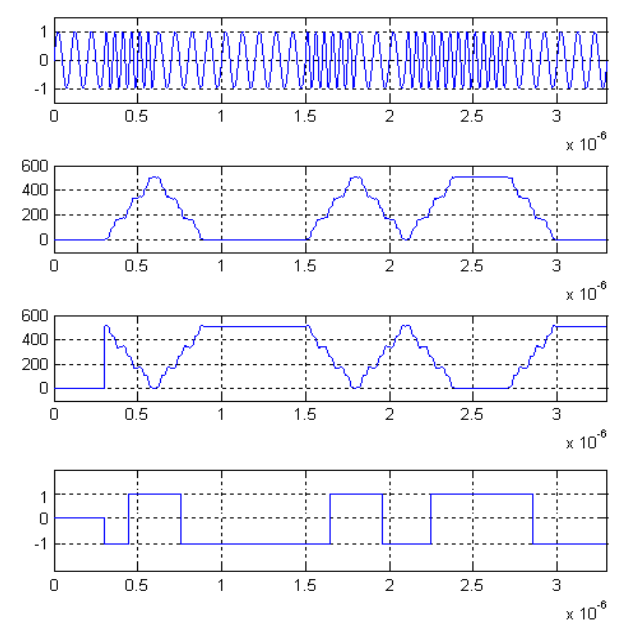

Fig. 5. 2-FSK demodulation process

\section{Conclusions}

This algorithm is realized based on the improvement of modern micro-electronic process integration and excellent high speed multiplier. It advances the non-storage of sample points which greatly saves the space of memory. And at the same time, this fast process with no-time-delay improves the utilization of the time domain.

But in calculation of big number, although the process optimizes the vertical vector of exponential matrix by periodicity and symmetrical, a large amount of calculation still should be done in $T_{s}$ cycle. A great challenge is that the tradeoff between the high performance and the short time. Therefore the calculation process and the calculation time percentage in $T_{s}$ are still two problems. They are also the directions of this algorithm development.

\section{References}

[1] Rogers.JAV, “ESM Processor Systems for High Pulse Density Radar Environments, ”IEEE Proc., Vol.132, Dec.1985.

[2] Liu,Guosui.Contrast experiment of ECCM of random to pseudorandom binary phase coded CW radar International Conference on Signal Processing Proceedings,ICSP v 2 Oct14-18 1996.

[3] Almeida L B. The fractional Fouriertrans formand time-frequency representations. IEEE Trans Signal Processing,1994,42(11):3084-3091.

[4] Raymond J. Lackey and Donald W.Upmal Speakeasy. The military software radio. IEEE communications magazine[J]1995.(5):56 61

[5] Robert Sgandurra. Digital Signal Processing Application Development: C6000 vs. PowerPC.Pentek,Inc.2002.2 